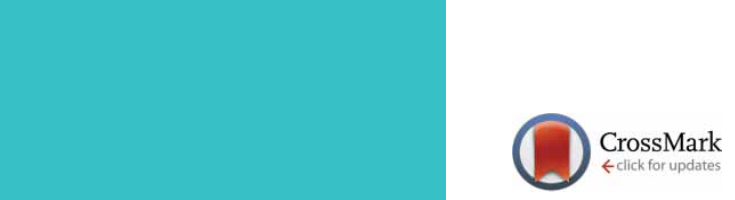

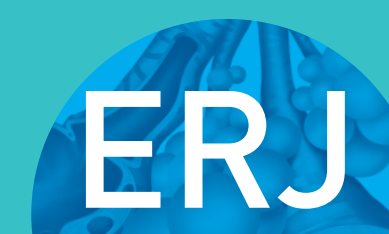

open research
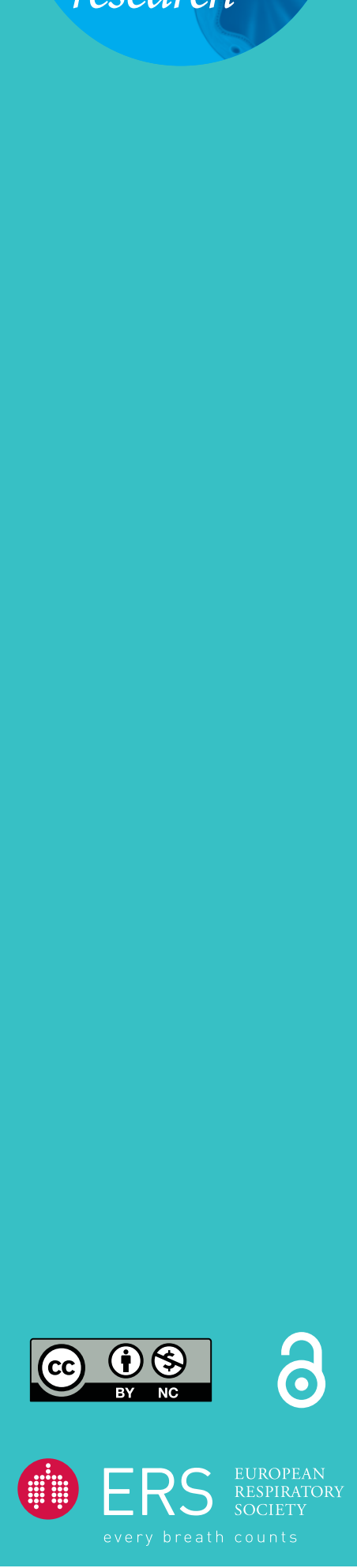

\section{Changing epidemiology of nontuberculous mycobacterial lung disease over the last two decades in a region of the Basque country}

\author{
Sandra Pedrero ${ }^{1}$, Eva Tabernero ${ }^{1}$, Eunate Arana-Arri ${ }^{2}$, Elena Urra ${ }^{3}$, \\ Maialen Larrea ${ }^{3}$ and Rafael Zalacain ${ }^{1}$
}

Affiliations: 'Dept of Respiratory Medicine, Hospital Universitario Cruces, Barakaldo, Spain. ${ }^{2}$ Research Unit, Hospital Universitario Cruces, Barakaldo, Spain. ${ }^{3}$ Dept of Microbiology, Hospital Universitario Cruces, Barakaldo, Spain.

Correspondence: Sandra Pedrero, Dept of Respiratory Medicine, Hospital Universitario Cruces, Plaza de Cruces, S/N, 48903 Barakaldo, Bizkaia, Spain. E-mail: sandra.pedrerotejadadosakidetza.eus

ABSTRACT Recent studies suggest an increasing prevalence of nontuberculous mycobacteria (NTM) lung disease. The aim of the present study was to describe incidence rates of NTM lung disease and trends therein in our area over a 20 -year period.

This was a retrospective study of all cases of NTM lung disease between 1997 and 2016 that met the 2007 American Thoracic Society criteria. We analysed the annual incidence rates, species of mycobacteria isolated, trends over time and annual mortality in 327 patients.

Mycobacterium kansasii was the most common mycobacterium isolated (84\%), followed by Mycobacterium avium complex (MAC) (13\%). We compared two periods: 1997-2006 (257 cases, 79\%) and 2007-2016 (70 cases, 21\%). The incidence rates tended to decrease across these years, with a peak of incidence in 2000 with 10.6 cases per 100000 . There was a clearly decreasing trend in M. kansasii infection, not only in the first period (incident rate ratio (IRR) $0.915,95 \%$ CI $0.88-0.90$; $\mathrm{p}<0.0001$ ) but also in the second (IRR 0.869, 95\% CI 0.780-1.014; $\mathrm{p}=0.080$ ), reaching 1.8 per 100000 in 2016. In contrast, MAC infection tended to increase across the two periods (IRR 1.251, 95\% CI 1.081-1.447; $\mathrm{p}=0.003)$.

In our region, the incidence of NTM lung disease has notably decreased in recent years. M. kansasii had high incidence rates in the first decade but clearly decreased in the second decade.

@ERSpublications

This epidemiological study shows a clear decrease in the incidence of nontuberculous mycobacterial lung disease, unlike other studies that have described an overall increase http://bit. ly/2ZH46nv

Cite this article as: Pedrero S, Tabernero E, Arana-Arri E, et al. Changing epidemiology of nontuberculous mycobacterial lung disease over the last two decades in a region of the Basque country. ERJ Open Res 2019; 5: 00110-2018 [https://doi.org/10.1183/23120541.00110-2018].

Received: 11 July 2018 | Accepted after revision: 2 Sept 2019

Copyright $\odot E R S$ 2019. This article is open access and distributed under the terms of the Creative Commons Attribution Non-Commercial Licence 4.0. 


\section{Introduction}

Lung disease due to nontuberculous mycobacteria (NTM), that is, environmental mycobacteria, is considered an emerging clinical entity [1]. Knowledge of this disease has improved thanks to advances in radiological and microbiological techniques, in particular, molecular identification methods, but there are still numerous uncertainties regarding both clinical management of the disease and the aetiopathogenic and other factors underlying the epidemiological differences between geographical areas.

Unlike tuberculosis, lung infection due to NTM is not a reportable disease, and hence, epidemiological data are not accurate, but most publications describe an increase in prevalence. On the other hand, diagnosis and epidemiological research are difficult as NTM are ubiquitous in the environment and their isolation in the laboratory does not necessarily imply disease [2, 3].

Environmental conditions and individual susceptibility play an important role in the prevalence of lung disease due to NTM, with areas of high disease risk and great variability in the causative species by geographical area. According to recent studies, the areas with the highest prevalence of the disease are regions with a high population density, a high level of education and a large proportion of the area covered by surface water with high evapotranspiration [4].

The importance of environmental mycobacteria lies in the fact that they require long antibiotic treatments with several drugs, generally for more than 1 year, with high associated costs and high toxicity [5]. Excess mortality has also been described in association with infection by NTM [6].

Thanks to the experience gained over 20 years in a tuberculosis control project at our hospital, communication and close cooperation between clinicians and the microbiology department have been strengthened in our daily work with scheduled information flow. Between 1997 and 2004, we detected a large number of cases of lung disease due to NTM, and since then, this condition has continued to be analysed.

The objective of this study was to describe the epidemiological pattern of lung disease due to NTM in our area, Bizkaia, and changes therein over the last 20 years. Bizkaia is a coastal and mountainous province of the Basque Country located in the north of Spain. It is an area with a high population density and is very industrialised, traditionally with chemical and metallurgical (especially steel) industries, though these have been progressively decreasing over the last 20 years. This area has not experienced significant population changes in the period studied.

\section{Methods}

A retrospective study was conducted including all cases of lung disease due to NTM that met the current American Thoracic Society (ATS) criteria in people over 18 years old diagnosed between 1997 and 2016 in Bizkaia. The epidemiological data were provided by the Microbiology Laboratory of Cruces University Hospital, which has a catchment population of 420000 . We analysed the annual incidence rates (IRs), species of mycobacteria isolated, trends in each species over time and annual mortality.

The inclusion criteria used for defining a case as "lung disease" were those established by the ATS in 2007 [3]. That is, we included all patients with mycobacterium isolates who met clinical, radiological and bacteriological criteria as follows: 1) presence of compatible symptoms (cough, fever, weight loss, haemoptysis or dyspnoea) not attributable to other diseases; 2) characteristic findings on chest radiography (infiltrate, nodules and cavitation) or high-resolution computed tomography scan (multifocal bronchiectasis with multiple nodules); and 3) positive culture results from at least two separate expectorated sputum samples or one bronchial wash or lavage or lung biopsy sample. Extrapulmonary samples were not considered. We excluded patients with a single isolate or who had no symptoms or radiological signs. Radiological and clinical data were obtained from the unified electronic health record, which extends back to 1995 .

\section{Microbiological analysis}

The methods for identifying mycobacteria have changed substantially over the last two decades, except for culture techniques (BACTEC MGIT 960 and Lowestein-Jensen medium) that have continued to be used throughout the study period. In the first 10 years, phenotypic methods were used, involving the assessment of growth rates and optimal temperatures, pigment production, colony morphology and biochemical features. In contrast, in recent years of the study period, other techniques have been adopted such as nucleic acid probes (AccuProbe, Gen-Probe); hsp65 gene PCR amplification followed by restriction fragment length polymorphism analysis using BstEII and HaeIII enzymes; agarose gel electrophoresis (PCR restriction fragment length polymorphism assays); analysis of the sequence of the hsp 65 fragment; line probe assays (GenoType, Hain Lifescience, used since 2015); and matrix-assisted laser desorption/ ionisation time-of-flight mass spectrometry (MALDI-TOF, used since 2014). 


\section{Statistical analysis}

The inter- and intra-group comparisons were carried out using parametric or nonparametric tests depending on the characteristics of the data distribution. Qualitative variables expressed as proportions or percentages were compared with Chi-squared or Fisher's exact test. For perioperative and follow-up variables, means were compared with t-tests if data were normally distributed, and otherwise, with the Mann-Whitney U-test. The IR for each mycobacteria species was calculated as the number of new cases divided by the number of people at risk per 100000 per year for the period 1997-2016. A Poisson regression model was used to analyse the incidence rate ratios (IRRs) by species and period of time included in the model, as well as to estimate temporal trends in the overall IRs. Results are reported as frequency rates $(95 \% \mathrm{CI})$. A p-value $\leqslant 0.05$ was considered statistically significant. Statistical analysis was carried out using IBM SPSS Statistics for Windows version 23.0 and STATA 13.

\section{Ethical considerations}

The study complied with the principles of the latest version of the Declaration of Helsinki and international regulations. Measures were taken to ensure the confidentiality and security of personal information concerning research participants.

\section{Results}

A total of 327 patients were diagnosed with NTM lung disease during the 20 years studied: 274 cases were attributed to M. kansasii (83.8\%), 43 to M. avium complex (13.1\%), and 10 to other mycobacteria such as M. xenopi, M. celatum, and M. abscessus (3.1\%). Any patients with a diagnosis of infection by M. avium complex, or specifically $M$. intracellulare, M. avium or M. chimaera, were classified as infected by MAC. All the patients met ATS diagnostic criteria for NTM lung disease and $85.6 \%$ of them were treated $(89.4 \%$ of cases of M. kansasii and $58.1 \%$ of cases of M. avium complex).

The overall IRs of lung disease due to NTM over the 20 years of study are shown in figure 1 . The highest IRs were recorded in 2000 and 2001, with 10.6 and 8.8 cases per 100000 , respectively. It can be observed that the IRs significantly decreased over the years to 1.8 cases per 100000 in 2016. In this context, the high IRs during the first few years of the study are striking. Hence, we decided to compare two 10-year periods, after observing a very marked decrease in the first decade, compared with the decrease in the second decade. The first decade from 1997 to 2006, in which there were 257 cases (79\%), and the second, from 2007 to 2016, in which there were 70 cases (21\%). We confirmed a decreasing trend in both decades, with a yearly average decrease of $6.4 \%$ (IRR $=0.939,95 \%$ CI $0.899-0.980 ; p=0.004)$ in the first decade, and of $6.2 \%(I R R=0.920,95 \%$ CI $0.849-0.997 ; \mathrm{p}=0.043)$ in the second decade.

Regarding the species involved, there was a clearly decreasing trend in $M$. kansasii over the course of the period studied, with an overall IRR of 0.858 (95\% CI=0.835-0.881; $\mathrm{p}<0.0001)$ and an annual decrease of $6.5 \%$. In contrast, there was a significant increasing trend in MAC in both decades, with an overall IRR of $1.133(95 \% \mathrm{CI}=1.058-1.213 ; \mathrm{p}<0.0001)$ and an annual rise of $3.3 \%$ (figure 2 ).

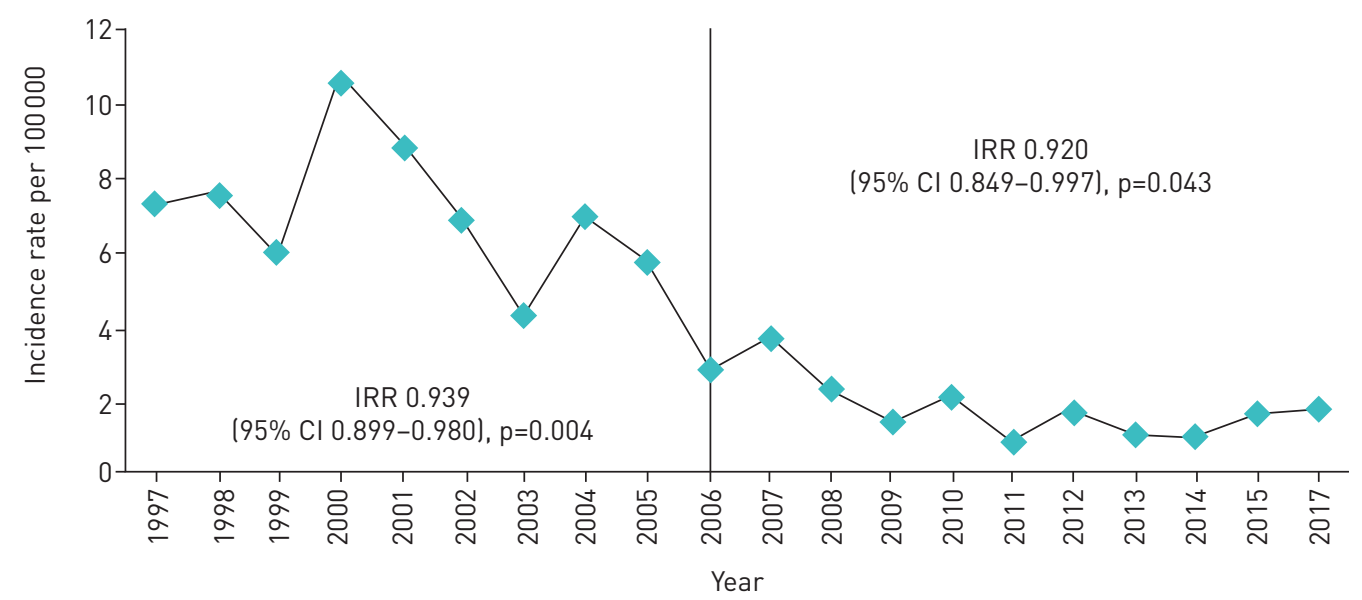

FIGURE 1 Overall incidence rates per 100000 population of lung disease due to nontuberculous mycobacteria between 1997 and 2016. IRR: incident rate ratio. 


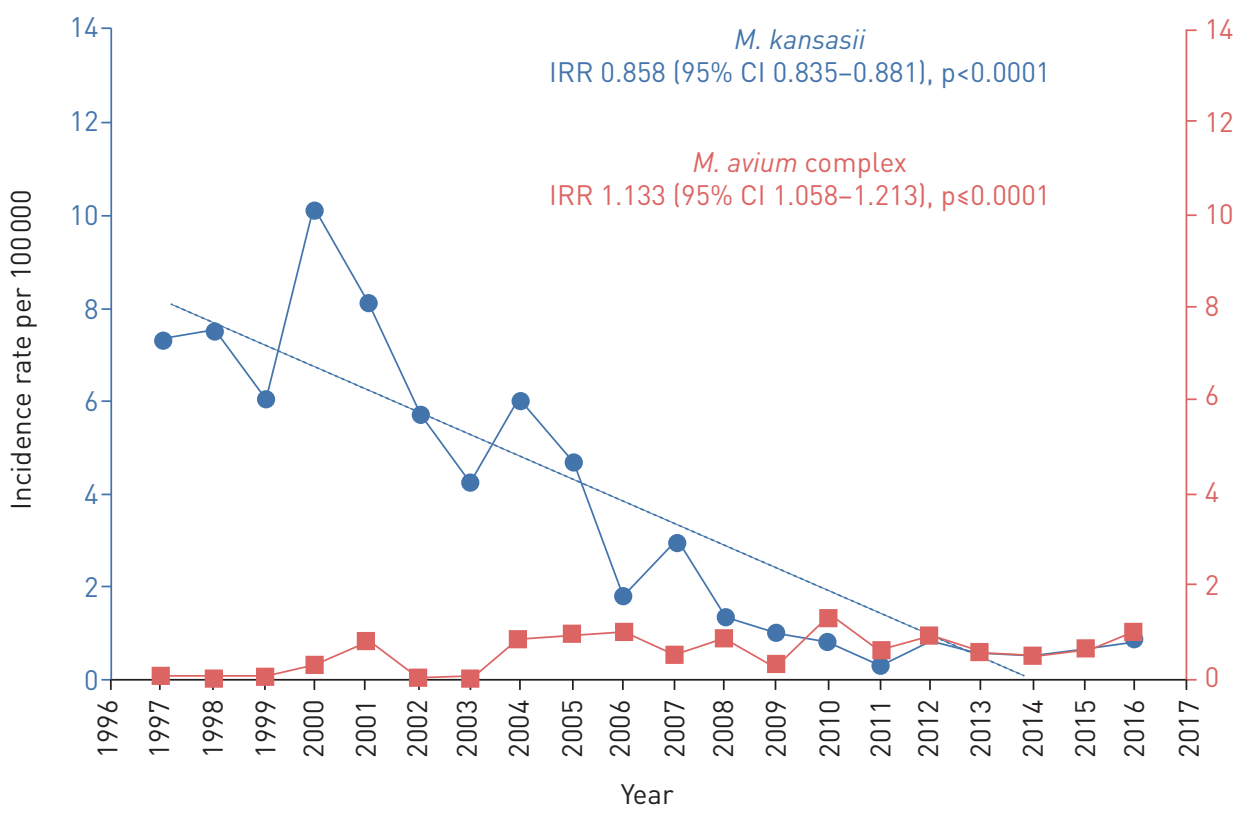

FIGURE 2 Incidence rates of lung disease due to nontuberculous mycobacteria between 1997 and 2016 by species involved: Mycobacterium kansasii and Mycobacterium avium complex. IRR: incident rate ratio.

Most patients with lung disease due to NTM were male (71.2\%) and the mean age overall was 56 years old. Notably, in the first decade, patients were slightly younger (mean: 54 years versus 61 years in the second decade, $\mathrm{p}=0.01)$ and more likely to be male $(75.2 \%$ versus $57 \%$ in the second decade, $\mathrm{p}=0.03)$ (table 1 ).

Concerning risk factors, no differences were found between the two decades in HIV, chronic obstructive pulmonary disease, history of lung disease, use of oral corticoids, alcohol use, liver disease, cancer or gastroesophageal reflux. On the other hand, the rate of active smoking was significantly higher in the first than the second decade ( $60.4 \%$ versus $44.8 \%$; $\mathrm{p}=0.003)$. Among radiological features, cavitation was the most common finding, especially in the first period, but no differences in radiological findings between the periods reached significance. Other findings included bronchiectasis, nodules and the tree-in-bud pattern. Notably, involvement was unilateral in almost half of the patients and overall upper lung lobes were the most common site of infection (table 2).

The overall annual mortality was $9.8 \%$ and the mortality attributed to mycobacteriosis was $1.8 \%$, with no significant differences between study periods. Nevertheless, the microbiological cure rate was higher in the first decade $(88.7 \%$ versus $70.1 \%$ in the second decade, $\mathrm{p}<0.001)$ (table 3 ).

Analysing these data by species, we have noticed differences between M. kansasii and M. avium complex over the period studied. IRs per 100000 population are shown in figure 2 by species. Concerning risk factors, no differences were found between NTM species except for the rates of active smoking and cancer,

\begin{tabular}{|c|c|c|c|c|}
\hline & Total & 1st decade ${ }^{\#}$ & $2^{\text {nd }}$ decade $e^{\pi}$ & $p$-value \\
\hline Patients with NTM lung disease & 327 & $257(78.6 \%)$ & $70(21.4 \%)$ & $<0.001$ \\
\hline \multicolumn{5}{|l|}{ NTM species } \\
\hline Mycobacterium kansasii & $274(83.8 \%)$ & $236(91.6 \%)$ & $38(54.3 \%)$ & $<0.001$ \\
\hline Mycobacterium avium complex & $43(13.1 \%)$ & $15(5.8 \%)$ & $28(40.0 \%)$ & \\
\hline Others $^{+}$ & $10(3.1 \%)$ & $6(1.8 \%)$ & $4(1.2 \%)$ & \\
\hline Age years mean & $56.8 \pm 18$ & $54 \pm 18.6$ & $61 \pm 16.9$ & 0.010 \\
\hline Male & $71.2 \%$ & $75.2 \%$ & $57.1 \%$ & 0.003 \\
\hline
\end{tabular}




\begin{tabular}{|c|c|c|c|c|}
\hline & Total & 1st decade $\#$ & 2nd decadeף & p-value \\
\hline \multicolumn{5}{|l|}{ Risk factors } \\
\hline HIV & $12.2 \%$ & $13.2 \%$ & $8.6 \%$ & 0.200 \\
\hline Smoking & $53.6 \%$ & $60.4 \%$ & $44.8 \%$ & 0.003 \\
\hline History of lung disease & $56 \%$ & $53 \%$ & $60.6 \%$ & 0.201 \\
\hline COPD & $30.1 \%$ & $34 \%$ & $23.8 \%$ & 0.113 \\
\hline Oral corticosteroid use & $6.7 \%$ & $8.2 \%$ & $4.5 \%$ & 0.283 \\
\hline Alcohol use & $18.3 \%$ & $20.2 \%$ & $15.4 \%$ & 0.285 \\
\hline Liver disease & $10.1 \%$ & $9.5 \%$ & $11.1 \%$ & 0.468 \\
\hline Cancer & $22.6 \%$ & $23.8 \%$ & $20.6 \%$ & 0.395 \\
\hline Gastroesophageal reflux & $9.6 \%$ & $11.5 \%$ & $6.6 \%$ & 0.233 \\
\hline \multicolumn{5}{|l|}{ Signs and symptoms } \\
\hline Cough/expectoration & $73.2 \%$ & $75.3 \%$ & $70.3 \%$ & 0.308 \\
\hline Fever & $40.4 \%$ & $42.5 \%$ & $37.3 \%$ & 0.323 \\
\hline Asthenia & $38 \%$ & $36.1 \%$ & $40.7 \%$ & 0.354 \\
\hline Weight loss & $24.1 \%$ & $23.8 \%$ & $24.6 \%$ & 0.484 \\
\hline Haemoptysis & $25.7 \%$ & $25 \%$ & $26.7 \%$ & 0.484 \\
\hline \multicolumn{5}{|l|}{ Radiological findings } \\
\hline Cavitation & $37.9 \%$ & $41.9 \%$ & $32.8 \%$ & 0.165 \\
\hline Bronchiectasis & $29.3 \%$ & $22.6 \%$ & $38.1 \%$ & 0.032 \\
\hline Nodules & $28.4 \%$ & $20.2 \%$ & $39.1 \%$ & 0.010 \\
\hline Tree-in-bud pattern & $25.7 \%$ & $15.7 \%$ & $39.3 \%$ & 0.001 \\
\hline \multicolumn{5}{|l|}{ Lung involvement } \\
\hline Unilateral & $45.7 \%$ & $49.4 \%$ & $40.9 \%$ & 0.547 \\
\hline Upper lung lobes & $52.4 \%$ & $58.8 \%$ & $43.3 \%$ & 0.260 \\
\hline Middle lung lobes & $15.2 \%$ & $11.8 \%$ & $20 \%$ & 0.260 \\
\hline Lower lung lobes & $15.2 \%$ & $9.4 \%$ & $6.7 \%$ & 0.260 \\
\hline Multifocal & $22.1 \%$ & $18.8 \%$ & $26.7 \%$ & 0.260 \\
\hline
\end{tabular}

which were significantly higher in the $M$. kansasii group $(63.8 \%$ versus $31.0 \%$; $\mathrm{p}<0.0001$, and $27.7 \%$ versus 9.5\%; p 0.011, respectively) (table 4).

Among radiological features, $M$. kansasii was associated with more cavitation, multifocal affectation and upper lobe predominance. On the other hand, M. avium complex was associated with bronchiectasis, nodules and tree-in-bud infiltrates with middle/lower lobe predominance and unilateral involvement. Microbiological cure rates were higher among patients with M. kansasii than MAC disease (89.0\% versus $76.7 \%, \mathrm{p}=0.029$ ), with a lower overall 1-year mortality, including NTM-related and other-cause mortality.

\section{Discussion}

The epidemiological data from our hospital show a clear decrease in the incidence of mycobacteriosis, especially since 2005, unlike other studies that have described a general increase in the incidence of this condition [7, 8]. In a study in Queensland, Australia, the incidence was observed to have increased from 2.2 cases per 100000 in 1999 to 3.2 cases per 100000 in 2005 [9]. Subsequently, in Oregon, USA, the

TABLE 3 Disease course and mortality from lung infections by nontuberculous mycobacteria (NTM), overall and in each decade

\begin{tabular}{lcccc} 
Disease course and mortality & Total & 1st decade & 2nd decade & p-value \\
\hline Microbiological cure & $275(84.1 \%)$ & $228(88.7 \%)$ & $47(70.1 \%)$ & $<0.0001$ \\
Overall mortality & $32(9.8 \%)$ & $22(8.5 \%)$ & $10(14.3 \%)$ & 0.153 \\
Deaths due to NTM lung disease & $6(1.8 \%)$ & $2(0.8 \%)$ & $4(5.7 \%)$ & 0.057 \\
Deaths due to other causes & $26(79.5 \%)$ & $20(7.7 \%)$ & $6(8.6 \%)$ & 0.339 \\
\hline \#: 1997-2006; ": $2007-2016$. & & & \\
\hline
\end{tabular}


TABLE 4 Comparison between Mycobacterium kansasii and Mycobacterium avium complex

\begin{tabular}{|c|c|c|c|c|}
\hline & Total & M. kansasii & M. avium complex & p-value \\
\hline Patients with NTM lung disease & 317 & 274 & 43 & \\
\hline \multicolumn{5}{|l|}{ Age years } \\
\hline Mean \pm SD & $58.5 \pm 17.8$ & $54.9 \pm 17.9$ & $63.0 \pm 17.3$ & 0.010 \\
\hline Median (IQR) & $61(44-73)$ & $57(42-69)$ & $68(47-78)$ & \\
\hline Male patients & $296(70.8 \%)$ & $204(75.0 \%)$ & $65(60.2 \%)$ & 0.003 \\
\hline \multicolumn{5}{|l|}{ Risk factors } \\
\hline HIV & $12.3 \%$ & $11.3 \%$ & $18.6 \%$ & 0.136 \\
\hline Smoking & $55.1 \%$ & $63.8 \%$ & $31.0 \%$ & $<0.0001$ \\
\hline History of lung disease & $55.8 \%$ & $53.9 \%$ & $61.0 \%$ & 0.276 \\
\hline COPD & $32.0 \%$ & $34.2 \%$ & $26.2 \%$ & 0.226 \\
\hline Oral corticosteroid use & $6.5 \%$ & $4.5 \%$ & $11.9 \%$ & 0.100 \\
\hline Alcohol use & $18.7 \%$ & $21.9 \%$ & $9.8 \%$ & 0.065 \\
\hline Liver disease & $10.7 \%$ & $9.3 \%$ & $14.6 \%$ & 0.252 \\
\hline Cancer & $22.7 \%$ & $27.7 \%$ & $9.5 \%$ & 0.011 \\
\hline Gastroesophageal reflux & $10.2 \%$ & $10.1 \%$ & $10.5 \%$ & 0.577 \\
\hline \multicolumn{5}{|l|}{ Signs and symptoms } \\
\hline Cough/expectoration & $73.6 \%$ & $70.9 \%$ & $80.5 \%$ & 0.166 \\
\hline Fever & $42.3 \%$ & $39.6 \%$ & $48.8 \%$ & 0.209 \\
\hline Asthenia & $37.6 \%$ & $34.4 \%$ & $45.0 \%$ & 0.168 \\
\hline Weight loss & $25.0 \%$ & $22.8 \%$ & $30.0 \%$ & 0.253 \\
\hline Haemoptysis & $26.7 \%$ & $30.2 \%$ & $17.9 \%$ & 0.105 \\
\hline \multicolumn{5}{|l|}{ Radiology } \\
\hline Cavitation & $38.2 \%$ & $48.1 \%$ & $12.5 \%$ & $<0.0001$ \\
\hline Bronchiectasis & $29.0 \%$ & $19.2 \%$ & $53.8 \%$ & $<0.0001$ \\
\hline Nodules & $26.6 \%$ & $22.2 \%$ & $37.5 \%$ & 0.053 \\
\hline Tree-in-bud pattern & $25.0 \%$ & $20.6 \%$ & $35.9 \%$ & 0.052 \\
\hline Lung involvement ${ }^{\#}$ & $49.7 \%$ & $42.7 \%$ & $67.5 \%$ & 0.003 \\
\hline \multicolumn{5}{|l|}{ Localisation } \\
\hline Upper lung lobes & $53.4 \%$ & $57.1 \%$ & $42.9 \%$ & 0044 \\
\hline Middle lung lobes & $15.0 \%$ & $10.2 \%$ & $28.6 \%$ & 0.044 \\
\hline Lower lung lobes & $8.3 \%$ & $7.1 \%$ & $11.4 \%$ & 0044 \\
\hline Multifocal & $23.3 \%$ & $25.5 \%$ & $17.1 \%$ & 0.044 \\
\hline \multicolumn{5}{|l|}{ Disease course and mortality } \\
\hline Microbiological cure & $87.3 \%$ & $89.0 \%$ & $76.7 \%$ & 0.029 \\
\hline Overall mortality & $9.8 \%$ & $9.1 \%$ & $14.0 \%$ & 0.229 \\
\hline Deaths due to NTM lung disease & $1.9 \%$ & $1.5 \%$ & $4.7 \%$ & 0.189 \\
\hline Deaths due to other causes & $7.9 \%$ & $7.7 \%$ & $9.3 \%$ & 0.448 \\
\hline
\end{tabular}

incidence of lung disease due to NTM was found to have increased from 4.8 cases per 100000 in 2007 to 5.6 per 100000 in 2012 [10], while over the same period, Shah et al. [11] observed an increase in pulmonary NTM isolates from 4.0 cases per 100000 in 2007 to 6.1 cases per 100000 in 2012 in England, Wales and Northern Ireland.

Our results also differ from those of a recent study in Asturias, Spain, which described a 35\% increase in the number of NTM isolates over a period of 8 years, although there were no changes in the number of patients with clinically significant NTM disease [12]. The only study we have found in which, as in ours, no increase was observed in the incidence of NTM was conducted in Denmark [13].

We should emphasise that the incidence of lung disease due to NTM varies across studies due to differences in the study design, samples used, diagnostic criteria applied and classification of lung disease due to NTM versus colonisation, these differences making it difficult to compare studies [3]. Additionally, numerous publications report numbers of isolates and not cases of disease. In our series, both the number of isolates and the number of cases of lung disease due to NTM have tended to decrease over the years.

The highest rates of NTM infection were found in 2000 with 10.6 per 100000 per year, decreasing to 1.8 per 100000 per year in 2016. The incidence of lung disease due to $M$. kansasii, the leading causative agent of NTM, decreased over the study period, with 236 patients diagnosed with this disease (91.6\%) in the first decade, and only 38 (54.3\%) in the second period. This peak in incidence in 2005 was described 
previously by a research group studying an overlapping geographical area [14], who attributed it to several factors yet to be demonstrated. In particular, the construction work for the underground train network (Metro Bilbao) might have led to there being more mycobacteria in the environment, while another nonexclusive explanation would be a change in water treatment, with more permissive temperatures and changes in water chlorination [15]. In this context, it has been stated that the main environmental niche for $M$. intracellulare appears to be in biofilms lining drinking water pipes and $M$. abscessus and $M$. kansasii have also been isolated from drinking water [9]. Another interesting possibility is that the high prevalence of $M$. kansasii coincided with a period of heavy industry, as has been also described in other studies $[16,18-]$.

Notably, in our study, despite the inclusion of individuals with structural lung damage who are more susceptible to infection, such as patients with cystic fibrosis or treated with immunosuppressive drugs (anti-tumour necrosis factor agents), and improvements in microbiological techniques in our hospital, we have not observed an increase in the prevalence of the disease. Indeed, NTM are considered emerging pathogens in patients with cystic fibrosis [19] and patients treated with anti-tumour necrosis factor agents [20], potentially causing significant morbidity and mortality.

Despite microbiological improvements, the increases observed in the detection of mycobacterial diseases do not coincide with the introduction of new microbiological techniques. In fact, newer techniques such as line probe assays (GenoType) and MALDI-TOF were introduced in 2014 and 2015 respectively, while the peaks of maximum incidence of NTM pulmonary disease were seen in 2000 and 2001.

On the other hand, this can be explained by the fact that in countries such as Spain, where the tuberculosis incidence is decreasing, the number of requested mycobacterial cultures also decreases and the awareness of mycobacterial disease as a differential diagnosis. In fact, in our study a decrease observed in the number of samples sent to the microbiology service was attributable to lower rates of suspicion of tuberculosis by clinicians. Specifically, the number of requests fell in the second decade to almost half (55\%) that in the first decade of the study and this coincided with the decrease in cases of mycobacterial disease.

To our knowledge, this is the first study that indicates such a clear decrease in the incidence rate of mycobacterial infection, though it is important to emphasise that this is due to a decrease in M. kansasii specifically in our area. It is possible that the pattern observed between 1997 and 2004 in our setting was due to higher exposure to $M$. kansasii, the load subsequently decreasing; this underlines the potential importance of exposure to high loads of environmental mycobacteria in the aetiopathogenesis and development of lung disease due to NTM.

As noted in the Introduction, there is marked geographical variability in the species isolated, with differences even within the same country, considering coastal or inland areas [18]. In a recent study in 30 countries across six continents, the most commonly isolated pathogen was MAC [21], with M. kansasii being the sixth most isolated species, with higher rates in eastern Europe and the UK, as well as in France, in the centre of Paris, and South America. The leading causative agent of the disease in our region was M. kansasii, consistent with the findings of earlier research in Spain based on a small sample in which M. kansasii was the most commonly isolated species [22]. In contrast, in a more recent study in a region close to our study area, also coastal, the most common causative agent of NTM was MAC [12].

Similarly, a trend towards increasing MAC and decreasing M. kansasii was shown in a large multicentre study [23] and in a recent study in Spain [24], where the authors described a decline in M. kansasii infections progressively from the early 2000s onwards, MAC becoming the most frequently isolated mycobacteria. Nevertheless, in both studies, the total number of isolates increased.

As in other studies, lung disease due to NTM is common in patients with HIV, in whom these types of lung infections were first described [25]. Nevertheless, such infections are affecting a growing number of people without HIV (87.8\% of patients in our series), this confirming the change in patient clinical profile noted previously [26, 27]. Patients who are immunocompetent commonly have underlying chronic conditions such as chronic obstructive pulmonary disease, a history of tuberculosis, a smoking habit [28] and/or other risk factors such as oral corticosteroid intake, alcohol use, liver disease, cancer and/or gastroesophageal reflux. In our series, it is notable that $M$. kansasii lung infections were significantly more likely to be in men and young people, which could be related to exposure to heavy industry.

Previous studies have demonstrated high rates of death due to NTM lung disease, but none have compared outcomes with matched population-based controls. Generally, research on mortality from this type of infection and associated factors is limited. Some studies have analysed overall 5-year mortality with very mixed findings, from $18 \%$ in a study from the USA [6] to $40 \%$ in Denmark [28]. More specifically, the aforementioned overall rate in Denmark was obtained in a comprehensive population-based study that 
found death rates of $39.7 \%$ due to MAC lung disease and $51 \%$ for $M$. xenopi disease [28], while rates of $36.3 \%$ and $43.2 \%$ respectively were found in Ontario, Canada [29]. In our study, we found an overall 1 -year mortality rate of $9.8 \%$, and though the mortality rate was slightly higher in the second period of study ( $14.3 \%$ versus $8.5 \%$ in the first period), the difference was not significant. As in other studies, we found low rates of mortality associated specifically with mycobacteria, it being difficult to establish the cause of death due to the presence of comorbidities [28, 29].

It is plausible that, in our study, the differences in radiological and microbiological cure rates between the first and second period can be explained by the shift in the predominant NTM species, with M. kansasii being the most prevalent species in the first decade with higher cure rates.

\section{Limitations}

This was a retrospective study with the disadvantages inherent to this type of study, in particular, concerning the quality of the data recorded for some clinical variables. Moreover, no information was gathered on the occupational history of the patients. Further, this study was carried out in the specific geographical area of Bizkaia, and hence, results cannot be extrapolated to other areas.

\section{Conclusions}

In our region, the incidence of lung disease due to NTM has notably decreased in recent years with a shift in the predominant NTM species. The mycobacteria most commonly found in our region was M. kansasii with IRs that were high in the first decade of the study and tended to decrease in the second decade. Mycobacteriosis-associated mortality was low with high cure rates, especially in the first decade of study. Although our study is not able to confirm this, we believe that greater environmental exposure, as well as host susceptibility, may have contributed to the development of this condition.

Conflict of interest: None declared.

\section{References}

1 Stout JE, Koh WK, Yew WW. Update on pulmonary disease due to non-tuberculous mycobacteria. Int J of Infect Dis 2016; 45: 123-134.

2 Larsson LO, Polverino E, Hoefsloot W, et al. Pulmonary disease by non-tuberculous mycobacteria-clinical management, unmet needs and future perspectives. Expert Rev Respir Med 2017; 11: 977-989.

3 Griffith DE, Aksamit T, Brown-Elliott BA, et al. An official ATS/IDSA statement: diagnosis, treatment, and prevention of nontuberculous mycobacterial diseases. Am J Respir Crit Care Med 2007; 175: 367-416.

4 Adjemian J, Olivier KN, Seitz AE, et al. Spatial clusters of nontuberculous mycobacterial lung disease in the United States. Am J Respir Crit Care Med 2012; 186: 553-558.

5 Ballarino GJ, Olivier KN, Claypool RJ, et al. Pulmonary nontuberculous mycobacterial infections: antibiotic treatment and associated costs. Respir Med 2009; 103: 1448-1455.

6 Fleshner M, Olivier KN, Shaw PA, et al. Mortality among patients with pulmonary non-tuberculous mycobacteria disease. Int J Tuberc Lung Dis 2016; 20: 582-587.

7 Winthrop KL, McNelley E, Kendall B, et al. Pulmonary nontuberculous mycobacterial disease prevalence and clinical features: an emerging public health disease. Am J Respir Crit Care Med 2010; 182: 977-982.

8 Kim RD, Greenberg DE, Ehrmantraut ME, et al. Pulmonary nontuberculous mycobacterial disease: prospective study of a distinct pre-existing syndrome. Am J Respir Crit Care Med 2008; 178: 1066-1074.

9 Thomson RM, on behalf of the NTM working group at the Queensland TB Control Centre, Queensland Mycobacterial Reference Laboratory. Changing epidemiology of pulmonary nontuberculous mycobacteria infections. Emerg Infect Dis 2010; 16: 1576-1583.

10 Henkle E, Hedberg K, Schafer S, et al. Population-based incidence of pulmonary nontuberculous mycobacterial disease in Oregon 2007 to 2012. Ann Am Thorac Soc 2015; 12: 642-647.

11 Shah NM, Davidson JA, Anderson LF, et al. Pulmonary Mycobacterium avium-intracellulare is the main driver of the rise in non-tuberculous mycobacteria incidence in England, Wales and Northern Ireland, 2007-2012. BMC Infect Dis 2016; 16: 195.

12 Martinez S, Cano A, Sota LA, et al. Micobacterias no tuberculosas ¿una amenaza emergente? Arch Bronconeumol 2017; 53: 554-560.

13 Hermansen TS, Ravn P, Svensson E, et al. Nontuberculous mycobacteria in Denmark, incidence and clinical importance during the last quarter-century. Sci Rep 2017; 7: 6696.

14 Leal Arranz MV, Gaafar A, Unzaga Barañano MJ, et al. Clinical and epidemiological study of disease caused by Mycobacterium kansasii in the metropolitan area of Bilbao, Spain. Arch Bronconeumol 2005; 41: 189-196.

15 Donohue MJ, Mistry JH, Donohue JM, et al. Increased frequency of nontuberculous mycobacteria detection at potable water taps within the United States. Environ Sci Technol 2015; 49: 6127-6133.

16 Hamada S, Ito Y, Hirai T, et al. Impact of industrial structure and soil exposure on the regional variations in pulmonary nontuberculous mycobacterial disease prevalence. Int J Mycobacteriol Medknow Publications 2016; 5: $170-176$.

17 Isaac-Renton JL, Allen EA, Chao CW, et al. Isolation and geographic distribution of Mycobacterium other than M. tuberculosis in British Columbia, 1972-81. CMAJ 1985; 133: 573-576.

18 Corbett EL, Hay M, Churchyard GJ, et al. Mycobacterium kansasii and M. scrofulaceum isolates from HIV-negative South African gold miners: incidence, clinical significance and radiology. Int J Tuberc Lung Dis 1999; 3: 501-507. 
19 Martiniano SL, Nick JA, Daley CL. Nontuberculous mycobacterial infections in cystic fibrosis. Clin Chest Med 2016; 37: 83-96.

20 Winthrop KL, Chang E, Yamashita S, et al. Nontuberculous mycobacteria infections and anti-tumor necrosis factor-alpha therapy. Emerg Infect Dis 2009; 15: 1556-1561.

21 Hoefsloot W, van Ingen J, Andrejak C, et al. The geographic diversity of nontuberculous mycobacteria isolated from pulmonary samples: an NTM-NET collaborative study. Eur Respir J 2013; 42: 1604-1613.

22 Martínez Moragón E, Menéndez R, Marco V, et al. Enfermedad pulmonar por micobacterias ambientales oportunistas en pacientes sin infección por el virus de la inmunodefíciencia humana. Factores de riesgo, clínica, diagnóstico y evolución. Arch de Bronconeumol 1996; 32: 170-175.

23 Martín-Casabona N, Bahrmand AR, Bennedsen J, et al. Non-tuberculous mycobacteria: patterns of isolation. A multi-country retrospective survey. Int J Tuberc Lung Dis 2004; 8: 1186-1193.

24 Santin M, Barrabeig I, Malchair P, et al. Pulmonary infections with nontuberculous mycobacteria, Catalonia, Spain, 1994-2014. Emerg Infect Dis 2018; 24: 1091-1094.

25 Marinelli DL, Albelda SM, Williams TM, et al. NTM infection in AIDS. Radiology 1986; 160: 77-82.

26 Maureen Cassidy P, Hedberg K, Saulson A, et al. Nontuberculous mycobacterial disease prevalence and risk factors: a changing epidemiology. Clin Infect Dis 2009; 49: 124-129.

27 Prevots DR, Marras TK. Epidemiology of human pulmonary infection with nontuberculous mycobacteria: a review. Clin Chest Med 2015; 36: 13-34.

28 Andrejak C, Thomsen VO, Johansen IS, et al. Nontuberculous pulmonary mycobacteriosis in Denmark: incidence and prognostic factors. Am J Respir Crit Care Med 2010; 181: 514-521.

29 Marras TK, Campitelli MA, Lu H, et al. Pulmonary nontuberculous mycobacteria-associated deaths, Ontario, Canada, 2001-2013. Emerg Infect Dis 2017; 23: 468-476. 